\title{
Update on Healthcare-Associated Blood Stream Infections in Febrile Neutropenic Pediatric Oncology Patients
}

\author{
Hadir A. El-Mahallawy1*, Safaa Shawky Hassan', Mohamed El-Wakil2, Manar M. Moneer³, \\ Lobna Shalaby 4 \\ ${ }^{1}$ Clinical Pathology Department, National Cancer Institute, Cairo University, Cairo, Egypt \\ ${ }^{2}$ Clinical Oncology Department, Faculty of Medicine, Beni Suef University, Beni Suef, Egypt \\ ${ }^{3}$ Biostatistics and Cancer Epidemiology Department, National Cancer Institute, Cairo University, Cairo, Egypt \\ ${ }^{4}$ Pediatric Oncology Department, National Cancer Institute, Cairo University, Cairo, Egypt \\ Email: wwakilmohamed@yahoo.com
}

Received 5 May 2015; accepted 5 June 2015; published 9 June 2015

Copyright (C) 2015 by authors and Scientific Research Publishing Inc.

This work is licensed under the Creative Commons Attribution International License (CC BY).

http://creativecommons.org/licenses/by/4.0/

c) (i) Open Access

\begin{abstract}
Background: Continuous surveillance of pattern of blood stream infection is necessary in febrile neutropenia especially with the recent escalating trend in the management of pediatric cancer patients towards intensified regimens and with the increase in infections caused by resistant organisms limiting the choice of antibiotics. Aim: Monitoring if a change has occurred in pattern of blood stream infections (BSI) in febrile neutropenic (FN) pediatric cancer patients. Methods: Surveillance of FN episodes with positive BSI was prospectively monitored and compared to a previous surveillance in the same pediatric oncology unit. Results: A total of 232 BSI positive episodes were documented in 192 patients during a 6 months period. The results of recent surveillance analysis showed an increase in intensified regimens of chemotherapy, antimicrobial resistance, and prolonged duration of episodes when compared to previous surveillance, with a $p$ value of $<0.001$, 0.005 , and $<0.001$, respectively. There was an apparent decrease in the crude mortality but this was not statistically significant, $6 \%$ in 2011 and $10 \%$ in 2006 . Conclusion: The pattern of BSI at our institution is still inclining towards gram positive organisms but is showing a shift towards more antibiotic resistance and prolonged episodes.
\end{abstract}

\section{Keywords}

Blood Stream Infections (BSI), Febrile Neutropenia (FN), Multidrug Resistant Organism (MDRO)

\footnotetext{
${ }^{*}$ Corresponding author.
}

How to cite this paper: El-Mahallawy, H.A., Hassan, S.S., El-Wakil, M., Moneer, M.M. and Shalaby, L. (2015) Update on Healthcare-Associated Blood Stream Infections in Febrile Neutropenic Pediatric Oncology Patients. Journal of Cancer Therapy, 6, 504-510. http://dx.doi.org/10.4236/jct.2015.66054 


\section{Introduction}

Blood stream infections are significant causes of morbidity and mortality in hematology and oncology patients [1] [2]. Duration and intensity of neutropenia are important risk factors for bacteremia and poor outcomes in patients with cancer [3]. Hematology patients and hematopoietic stem cell transplant (HSCT) recipients undergoing intensive myelosuppressive or immunosuppressive treatment are at high risk for severe, life-threatening, bacterial infections. Thirteen to $60 \%$ of HSCT recipients develop BSI, which are associated with $12 \%$ to $42 \%$ mortality [4]. Monitoring local epidemiology is important in directing the strategy of management of febrile neutropenia. Based on the new evidence, international guidelines on antimicrobial prophylaxis have been recently updated, and targeted prophylaxis schedules have been proposed for different clinical settings [5].

Growing resistance to standard antibiotics leads to increased use of broad-spectrum regimens, including the selection of resistant pathogens, predisposition to fungal infections and Clostridium difficile-associated diarrhea [6] [7]. Building recommendations for empirical therapy in this era of growing resistance is challenging [8]. Therefore, we attempted in this study to monitor the changing epidemiology that might have occurred in the pattern of blood stream infections in febrile neutropenic pediatric oncology patients in our institution.

\section{Patients and Methods}

The study was composed of 2 parts. A prospective part including surveillance of pediatric cases with cancer receiving chemotherapy and developing bacteremia while febrile neutopenic and hospitalized in the period from April to September 2011 at the Pediatric Oncology Department of National Institute (NCI), Cairo University, Egypt. The other part was comparing the 2011 surveillance to a previous one recorded in 2006 at the same unit [7]. Patients who received a marrow transplant were not included in both cohorts. The study was approved by NCI Ethics Committee and an informed consent was obtained from parents of participants.

Febrile neutropenia is defined as an oral temperature $>38.5^{\circ} \mathrm{C}$ or two consecutive readings of $>38.0^{\circ} \mathrm{C}$ for 2 hours and an absolute neutrophil count $<0.5 \times 10^{9} / \mathrm{L}$, or expected to fall below $0.5 \times 10^{9} / \mathrm{L}$ [9].

Data collected at first day of fever included age, diagnosis, state of disease, absolute neutrophil count (ANC), clinically documented infections, previous febrile episodes treated with broad spectrum antibiotics within one month preceding the current episode. Other data included were type of chemotherapy either standardized or intensified protocols. Intensified protocols are the regimens used in induction therapy, while standardized protocols are those used for maintenance. Follow up of patients was carried out as regard antibiotic received, need of shift, duration of episode and outcome of patients one month following febrile neutropenic episode.

Microbiology. Two blood cultures sets were usually drawn from each patient within the first day of fever, one from peripheral veins, and the other from cannula site, portacath, or CVC. Bactec 9050 incubator was used. Blood culture sampling was the same used in the study performed in 2006 (7). Identification of isolates was carried out using Microscan dried gram negative MIC/Combo and dried gram positive MIC/Combo panels Seimens Healthcare Diagnostics Ltd (Sir William Siemens Sq. Frimley, Cambeley, UK GU16 8QD) for Gram-negative and Gram-positive organisms, respectively. The panel of antibiotics used for Gram negative included amikacin, amoxicillin-clavulanate, ampicillin-sulbactam, aztreonam, cefepime, cefoperazone, ceftazidime, cefuroxime, ciprofloxacin, gentamycin, imipenem, meropenem, norfloxacin, piperacillin, piperacillin-tazobactam, ticarcicilline-clavulanate and trimethoprim-sulfamethoxazole. Clindamycin, erythromycin, gatifloxacin, gentamycin, levofloxacin, linezolid, moxifloxacin, oxacillin, synercid, tetracycline, and vancomycin were the main antibiotics included in gram positive panels. Interpretation of susceptibility test results was done by referring to the CLSI guidelines [10]. A multidrug resistant organism (MDRO) was defined as acquired non-susceptibility to at least one agent in three or more antimicrobial categories [11]; which was the same definition used in the evaluation of the 2006 patient cohort.

Management. During the febrile episode patients were hospitalized and treated with double agent broad spectrum antimicrobial therapy. The empirical regimen of hospital guidelines were cefepime/amikacine (FEP/ AK), or piperacillin-tazobactem/amikacin (TZP/AK); with second line meropenem/amikacin (MEM/AK). Empirical antifungal was used if fever persisted till day 5. An antiviral (zovirax) was used when indicated. Antibiotic continued until patient became afebrile and ANC exceeded $0.5 \times 10^{9} \mathrm{~L}$. The episode was considered successfully controlled when fever and clinical signs resolved within 5 - 7 days. A prolonged episode was defined by persistent fever for more than 7 days, with other measures taken into consideration including the general condition of the patient as regards severity and duration of neutropenia, in addition to any associated clinical 
focus. Guidelines used in our institution were adjusted from the update outlined by the infectious diseases of America (IDSA) [12] taking into consideration the local epidemiology.

Statistical Methods. Data were analyzed using SPSSwin statistical package version 15. Chi-square or Fisher's Exact tests were used to compare qualitative variables. Comparison between two groups regarding numeric variables was done using either Student's t-test or Mann-Whitney test as appropriate. Multivariate analysis was done using binary logistic regression by forward conditional method. P-value $<0.05$ was considered significant. All tests were two tailed.

\section{Results}

Patients Characteristic. During 6 months period of 2011, 232 febrile bacteremic episodes were recorded in 192 patients. The patient age ranged from 1.5 month to 18 years with a mean age of $6.8 \pm 4.5$. They were 119 males and 73 females. Their diagnoses were acute lymphoblastic leukemia, acute myeloblastic leukemia, lymphoma, and solid tumors in 72 (37.5\%), 36 (18.7\%), 21 (11\%), and 63 (32.8\%), respectively.

Clinical Features. The mean value of fever at day 1 was $38.5^{\circ} \mathrm{C} \pm 0.5^{\circ} \mathrm{C}$. Clinical documented infections (CDI) were recorded in 114 (49\%) episodes, whereas, 118 (51\%) episodes lacked a focus. The sites of CDI were lower respiratory tract, diarrhea, or skin and intra-venous line related infections in 55 (24\%), 24 (10\%), and 14 (6\%), respectively. Other sites of infections included otitis media, tonsillitis, sinusitis, ophthalmic infection, herpetic lesions and chicken pox were reported in 68 (29\%) episodes. Previous febrile episodes with intake of empirical broad spectrum antibiotics were recorded in 142 (61\%) episode, while 90 (39\%) showed no previous febrile episode. Neutropenia was below $0.5 \times 10^{9} / \mathrm{L}$ in 163 episode (70\%) and extended for $\geq 7$ days in $122(53 \%)$ of episodes.

Microbiology. Gram-positive organisms (GPC) were the predominant causative agents of BSI, constituting 168 (72\%) of isolated organisms, while 56 (24\%) of BSIs were caused by Gram-negative organisms (GNR), and 8 (3\%) were mixed Infections. The organisms isolated are summarized in Table 1. Of the isolated pathogens, 119 (51\%) were MDRO. Comparison of percentage of multiresistance among some of the isolated organisms is illustrated in Figure 1.

Table 1. Isolated organisms from 232 Bacteremic febrile episodes in Pediatric Cancer patients in 2011.

\begin{tabular}{|c|c|}
\hline Organisms & Number (\%) \\
\hline Gram-positive organisms & $168(72.4)$ \\
\hline - Coagulase negative Staphylococci & $135(58.2)$ \\
\hline - Streptococcus spp. & 16 (6.9) (7 enterococci) \\
\hline - Stapylococcus aureus & 13 (5.6) (12 MRSA) \\
\hline - $\quad$ Micrococci & $1(0.4)$ \\
\hline - Mixed gram positive cocci & $3(1.3)$ \\
\hline Gram-negative organisms & $56(24.1)$ \\
\hline $\begin{array}{l}\text { - Non-fermenters } \\
\text { Acinetobacter sp. }\end{array}$ & $6(2.6)$ \\
\hline Pseudomonas sp. & $6(2.6)$ \\
\hline Stenotrophomonas maltophilia & $1(0.4)$ \\
\hline $\begin{array}{r}\text { - Enterobacteriacae } \\
\text { E. coli }\end{array}$ & $11(4.7)$ \\
\hline Klebsiella spp. & $16(6.9)$ \\
\hline Enterobacter spp. & $9(3.9)$ \\
\hline Salmonella spp. & $1(0.4)$ \\
\hline Citrobacter freundii & $1(0.4)$ \\
\hline Achromobacter sp & $3(1.3)$ \\
\hline $\begin{array}{r}-\quad \text { Other GNR } \\
\text { Alcaligenis }\end{array}$ & $2(0.9)$ \\
\hline Mixed growth & $8(3.4)$ \\
\hline
\end{tabular}




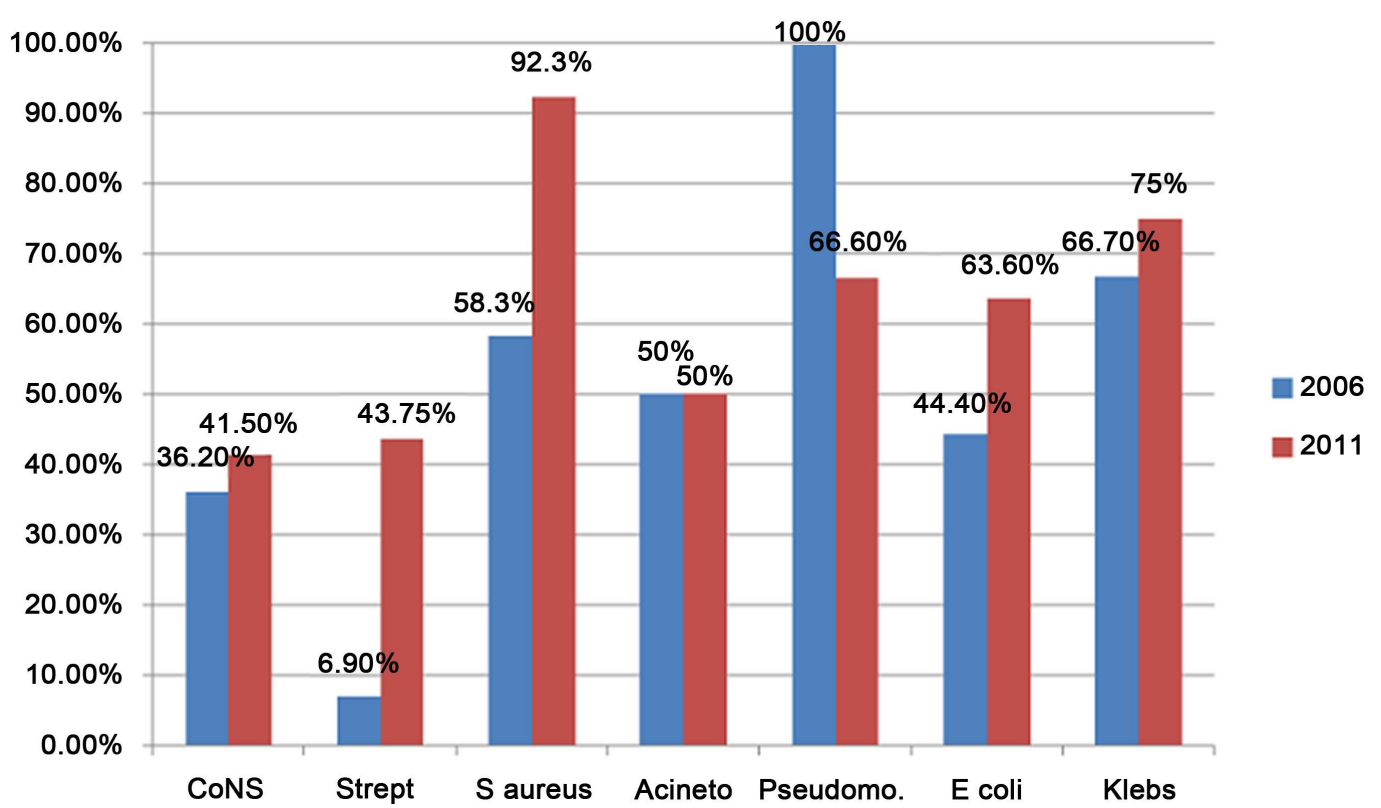

Figure 1. Comparison of percentage of multiresistance among isolated organisms. CoNS, (coagulase negative Staphylococci), Strept (Streptococcal sp.), S aureus (Staphylococcus aureus), Acineto (Acinetobacter sp.), Ps aerug (Pseudomonas aeruginosa), E. coli (Escherechia coli) Kleb (Klebsiells sp.) between the two periods in 2006 \& 2011.

Course and Outcome. Recovery within 7 days was documented in 87 (38\%) of the episodes, whereas the infectious episode extended for 7 days or more in 145 (62\%) of the cases. The need to shift primary line of antibiotic was recorded in 55 (24\%) episode, while vancomycin was added in 120 (52\%) episodes. An empirical antifungal was given in 126 (54\%) episode. Continued use of antifungals after 7 days were recorded in 104 episodes (45\%). Crude mortality, i.e. mortality occurring within one month of infection was recorded in 15/232 (6.5\%) patients; (8/168 (4.8\%) of the GPC, 12.5\%, 7/56 (12.5\%) of the GNR).

The results of comparison of analysis of surveillance of blood stream infections (BSI) between over 6 months duration in 2006 and 2011 are demonstrated in Table 2.

\section{Discussion}

With the increased use of intensified chemotherapy in the growing population of patients with malignant disorders, profound and prolonged neutropenia are expected to occur with the resultant increase in the incidence of infections accompanied by difficulties in their management. Therefore, we attempted to investigate the change of epidemiology in FN pediatric cancer patients receiving chemotherapy at National Cancer Institute, its effect on morbidity and mortality, and the effectiveness of supportive care as regards need of antibiotic shift, duration and outcome of infectious episodes.

Pattern of blood stream infections in febrile neutropenia has passed through several shifts through the previous decades necessitating continuous surveillance over cancer centers worldwide to detect changes in local epidemiology and modify management guidelines accordingly. In the current study, we compared between surveillance results of BSI in pediatric cancer patients during a six months period in the year 2006 \& six months in the year 2011; GPC constituted 75\% of organisms isolated in the year 2006 and 72\% in 2011. Whereas, gram negative pathogens were isolated in 25\% and 24\% of children with BSI in the years 2006 and 2011, respectively. In our institution, the pattern of BSI in pediatric cancer patients was characterized by predominance of gram positive bacteremia in several previous studies [7] [13]. In recent years, the etiology of BSI in neutropenic patients in some cancer centers has shifted from gram-positive to gram-negative organisms [14], but this is in adult population. It is evident that pediatric patients are still having predominance of GPC as causes of BSIs. In the present study, there was an observed variation in the relative frequency of organisms within total GNRs with a decrease in the non-fermenters from 61\% (36/59) in 2006 to 23\% (13/56) in 2011 and an increase in Enterobac- 
Table 2. Comparison between some parameters reported in surveillance analysis of blood stream infections in febrile neutropenic pediatric patients with cancer in $2011(\mathrm{~N}=192)$ and that done on $2006(\mathrm{~N}=193)$.

\begin{tabular}{|c|c|c|c|}
\hline Parameter & $2006 N=239 N(\%)$ & $2011 N=232 N(\%)$ & p-value \\
\hline Age (mean \pm SD) & $6.7 \pm 4.8$ & $6.8 \pm 4.5$ & 0.832 \\
\hline $\begin{array}{ll} & \text { Day } 7 \text { evaluation } \\
\text { - } & \text { Fever } \\
\text { - } & \text { Recovery }\end{array}$ & $\begin{array}{l}103(43) \\
136(57)\end{array}$ & $\begin{array}{c}145(62) \\
87(38)\end{array}$ & $<0.001$ \\
\hline $\begin{array}{ll} & \text { Chemotherapy } \\
\text { - } & \text { Standard } \\
\text { - } & \text { Intensified }\end{array}$ & $\begin{array}{l}129(54) \\
110(46)\end{array}$ & $\begin{array}{c}74(32) \\
158(68)\end{array}$ & $<0.001$ \\
\hline $\begin{array}{ll} & \text { Clinical infections } \\
\text { - } & \text { No } \\
\text { - } & \text { Yes }\end{array}$ & $\begin{array}{l}114(48) \\
125(52)\end{array}$ & $\begin{array}{l}118 \text { (51) } \\
114(49)\end{array}$ & 0.492 \\
\hline \begin{tabular}{ll} 
& \multicolumn{1}{c}{ Organism } \\
- & Gram positive cocci \\
- & Gram negative rods \\
- & Mixed
\end{tabular} & $\begin{array}{c}180(75) \\
59(25) \\
0(0)\end{array}$ & $\begin{array}{c}168(72) \\
56(24) \\
8(4)\end{array}$ & 0.010 \\
\hline $\begin{array}{ll} & \text { Antibiotic resistance } \\
\text { - } & \text { Sensitive } \\
\text { - } & \text { Resistant }\end{array}$ & $\begin{array}{c}147(62) \\
92(38)\end{array}$ & $\begin{array}{l}113(49) \\
119(51)\end{array}$ & 0.005 \\
\hline $\begin{array}{ll} & \text { Outcome } \\
\text { - } & \text { Alive } \\
\text { - } & \text { Dead }\end{array}$ & $\begin{array}{l}214(90) \\
25(10)\end{array}$ & $\begin{array}{c}217(94) \\
15(6)\end{array}$ & 0.120 \\
\hline
\end{tabular}

teriacae in 2011 (77\%) compared to 2006 (39\%). This finding might be due to dedication of a specified infection control team concerned with applying strict infection control measures in the pediatric unit.

Though there was no change in the pattern of pathogens causing BSIs in pediatric cancer patients in our institution, a significant increase in antimicrobial resistance was recorded. There was an increased incidence of resistance in 2011 compared to 2006 as MDRO was significantly higher in 2011 (51\%) than in 2006 (38.5\%) (p value 0.005 ). There is a growing problem of antimicrobial resistance among the pathogens isolated from hematology patients and hematopoietic stem cell transplant recipients in many centers, and this increasingly influences the choice of empirical therapy [6]. Previous intake of antibiotics in febrile neutropenic patients was reported to be the most frequent risk factor for BSI caused by MDROs [7] [15]. The increase of resistance recorded in the present study could be explained by that patients with hematological malignancies present with repeated febrile episodes and thus, receive repeated cycles of empirical antibiotics. Treatment options are very limited with increasing resistance, and the emergence of multiresistant Gram-negative organisms is inviting the use of old antibiotics, notably colistin/polymyxin B and fosfomycin 2 - 6 and tigecycline [16]. Similarly, the emergence of Gram-positive pathogens with reduced susceptibility to glycopeptides is leading to the use of linezolid, daptomycin and tigecycline in hematology patients [17]. Thus, modification of management guidelines is recommended in the era of growing resistance possibly by adopting a de-escalation strategy to avoid the collateral damage initiated by antibiotic overuse [6].

Going on with the recent advances in the management of hematological malignancies towards intensified chemotherapy, more intensified regimens were used in the pediatric oncology department of NCI in the year 2011 (68\%), compared to the year 2006 (46\%); $p=0.001$. This is expected to be reflected on indicators of neutropenia, as intensified protocols will be significantly associated with more profound and prolonged duration of neutropenia. The increased use of intensified regimens of chemotherapy is accompanied by longer episodes and longer duration of neutropenia. The increased use of intensified regimens of chemotherapy seems to be accompanied by longer episodes and longer duration of neutropenia. Thus, it is evident that neutropenia is still a major determinant indicator of risk in febrile neutropenia.

Despite advances in supportive care following cancer chemotherapy in recent practice, infections remain a major cause of morbidity, healthcare resource use and decline in efficacy as a result of delays in chemotherapy [9]. In the present study, an unfavorable outcome was encountered in 6\% (15/232) of the surveillance analysis of 
2011 compared to $10 \%$ in 2006. There was an apparent drop in mortality rate though it was not statistically different, yet the results are keeping on with the decreasing figures of mortality encountered for F\&N worldwide. In the same institution, infection attributable mortality in 100 pediatric patients with hematological malignancies was recorded to be 3\% [18]. Generally, the highest mortality is reported to be associated with GNRs, approaching a rate of $18 \%$ and $5 \%$ in GP bacteremias [9]. It was concluded that the low mortality rates reported over the last decade in pediatric oncology patients could be due to improved management of BSIs and due to advances in intensive care in this population of patients [19].

Thus, it could be concluded from the comparison of results of febrile neutropenia surveillance in one cancer center five years apart that the pattern of BSI at our institution is still inclining towards gram positive organisms but is showing a big shift towards more antibiotic resistance. The increase in frequency of fungal infection in our patients could be due to the increased use of intensified chemotherapeutic protocols. This increase in frequency of IFI necessitates a change in the management approach towards prevention and prophylaxis in high risk groups rather than waiting for infection to occur. An apparent decline in figures of mortality in our institution invites us to follow up closely the pattern of FN in pediatric cancer patients for better performance and continuous progression in the field of supportive care.

\section{Fund}

This study is done as part of work of NCI and is funded by NCI.

\section{Conflict}

No conflict of interest is considered for any of the authors of this study.

\section{References}

[1] Rodríguez, L., Ethier, M.C., Phillips, B., Lehrnbecher, T., Doyle, J. and Sung, L. (2012) Utility of Peripheral Blood Cultures in Patients with Cancer and Suspected Blood Stream Infections: A Systematic Review. Support Care Cancer, 20, 3261-3267. http://dx.doi.org/10.1007/s00520-012-1471-2

[2] Sancho, S., Artero, A., Zaragoza, R., Camarena, J.J., González, R. and Nogueira, J.M. (2012) Impact of Nosocomial Polymicrobial Bloodstream Infections on the Outcome in Critically Ill Patients. European Journal of Clinical Microbiology \& Infectious Diseases, 31, 1791-1796. http://dx.doi.org/10.1007/s10096-011-1503-8

[3] Samonis, G., Vardakas, K.Z., Maraki, S., Tansarli, G.S., Dimopoulou, D., Kofteridis, D.P., et al., (2013) A Prospective Study of Characteristics and Outcomes of Bacteremia in Patients with Solid Organ or Hematologic Malignancies. Support Care Cancer, 21, 2521. http://dx.doi.org/10.1007/s00520-013-1816-5

[4] Trecarichi, E.M., Tumbarello, M., Spanu, T., Caira, M., Fianchi, L., Chiusolo, P., et al. (2009) Incidence and Clinical Impact of Extended-Spectrum-Beta-Lactamase (ESBL) Production and Fluoroquinolone Resistance in Bloodstream Infections Caused by Escherichia coli in Patients with Hematological Malignancies. Journal of Infection, 58, 299-307. http://dx.doi.org/10.1016/j.jinf.2009.02.002

[5] Girmenia, C. and Menichetti, F. (2011) Current Epidemiology and Prevention of Infectious Complications in Cancer Patients. European Oncology \& Haematology, 7, 270-277.

[6] Averbuch, D., Orasch, C., Cordonnier, C., Livermore, D.M., Mikulska, M., Viscoli, C., et al. (2013) European Guidelines for Empirical Antibacterial Therapy for Febrile Neutropenic Patients in the Era of Emerging Resistance: Summary of the 4th European Conference on Infections in Leukemia. Haematologica, 98, 1826-1835. http://dx.doi.org/10.3324/haematol.2013.091025

[7] El-Mahallawy, H.A., El-Wakil, M., Moneer, M.M. and Shalaby, L. (2011) Antibiotic Resistance Is Associated With Longer Bacteremic Episodes and Worse Outcome in Febrile Neutropenic Children With Cancer. Pediatric Blood \& Cancer, 57, 238-288. http://dx.doi.org/10.1002/pbc.22926

[8] Giamarellou, H. (2010) Multidrug-Resistant Gram-Negative Bacteria: How to Treat and for How Long. International Journal of Antimicrobial Agents, 36, 50-54. http://dx.doi.org/10.1016/j.ijantimicag.2010.11.014

[9] Naurois, J., Novitzky, I., Basso, G.M.J, Marti, M., Cullen, M.H., Roila, F., et al., On Behalf of the ESMO Guidelines Working Group (2010) Management of Febrile Neutropenia: ESMO Clinical Practice Guidelines. Annals of Oncology, 21, 252-256.

[10] Clinical and Laboratory Standards Institute (2009) Performance Standards for Antimicrobial Susceptibility Testing. Nineteenth Informational Supplement M100-S19. Clinical and Laboratory Standards Institute, Wayne.

[11] Magiorakos, A.P., Srinivasan, A., Carey, R.B., Carmeli, Y., Falagas, M.E., Giske, C.G., et al. (2012) Multidrug-Resis- 
tant, Extensively Drug-Resistant and Pandrug-Resistant Bacteria: An International Expert Proposal for Interim Standard Definitions for Acquired Resistance. Clinical Microbiology and Infection, 18, 268-281. http://dx.doi.org/10.1111/j.1469-0691.2011.03570.x

[12] Freifeld, A., Bow, E., Sepkowitz, K., Boeckh, M., Ito, J., Mullen, C., Raad, I., Young, J. and Wingard, J. (2011) Clinical Practiceguideline for the Use Antimicrobial Agents in Neutropenic Patients with Cance: 2010 Update by the Infectious Disease Society of America. Clinical Infectious Diseases, 52, 56-93. http://dx.doi.org/10.1093/cid/cir073

[13] El-Mahallawy, H.A., Sidhom, I., El-Din, N.H., Zamzam, M. and El-Lamie, M.M. (2005) Clinical and Microbiological Determinants of Serious Blood Stream Infections in Egyptian Pediatric Cancer Patients: A One Year Study. International Journal of Infectious Diseases, 9, 43-51. http://dx.doi.org/10.1016/j.ijid.2003.11.010

[14] Gudiol, C., Bodro, M., Simonetti, A., Tubau, F., González-Barca, E., Cisnal, M., et al. (2013) Changing Aetiology, Clinical Features, Antimicrobial Resistance, and Outcomes of Bloodstream Infection in Neutropenic Cancer Patients .Clinical Microbiology and Infection, 19, 474-479. http://dx.doi.org/10.1111/j.1469-0691.2012.03879.x

[15] Mar, M., Carlota, G., Carol, G.-V., Carmen, A. and Jordi, C. (2014) Bloodstream Infections in Patients with Solid Tumors: Epidemiology, Antibiotic Therapy, and Outcomes in 528 Episodes in a Single Center. Medicine, 93, 143-149. http://dx.doi.org/10.1097/MD.0000000000000026

[16] Livermore, D.M., Warner, M., Mushtaq, S., Doumith, M., Zhang, J. and Woodford, N. (2011) What Remains against Carbapenem-Resistant Enterobacteriaceae? Evaluation of Chloramphenicol, Ciprofloxacin, Colistin, Fosfomycin, Minocycline, Nitrofurantoin, Temocillin and Tigecycline. International Journal of Antimicrobial Agents, 37, 415-419. http://dx.doi.org/10.1016/j.ijantimicag.2011.01.012

[17] Averbuch, D., Cordonnier, C., Livermore, D.M., Mikulska, M., Viscoli, C., Orasch, C., et al. (2013) Targeted Therapy against Multi-Resistant Bacteria in Leukemic and Hematopoietic Stem Cell Transplant Recipients: Guidelines of the 4th European Conference on Infections in Leukemia (ECIL-4, 2011). Haematologica, 98, 1836-1847. http://dx.doi.org/10.3324/haematol.2013.091330

[18] Sayed, H., El-Mahallawy, H., Kaddah, A., Ismael, H. and Talaat, S. (2009) Profiles of Infections in Newly Diagnosed Patients with Acute Leukemia during the Induction Phase of Treatment. Journal of the Egyptian National Cancer Institute, 21, 315-322.

[19] Simon, A., Ammann, R., Bode, U., Fleischhack, G., Wenchel, H., Schwamborn, D., Gravou, C., et al. (2008) Healthcare-Associated Infections in Pediatric Cancer Patients: Results of a Prospective Surveillance Study from University Hospitals in Germany and Switzerland. BMC Infectious Diseases, 8, 70-75. http://dx.doi.org/10.1186/1471-2334-8-70 